\title{
EVOLUCIÓN PALEOAMBIENTAL DURANTE EL TRÁNSITO CRETÁCICO/PALEÓGENO BASADA EN FORAMINÍFEROS BENTÓNICOS
}

\author{
Laia ALEGRET BADIOLA
}

Departamento Ciencias de la Tierra, Universidad de Zaragoza. 50009 Zaragoza. laia@unizar.es

Alegret Badiola, L. 2008. Evolución paleoambiental durante el tránsito Cretácico/Paleógeno basada en foraminíferos bentónicos. [Paleoenvironmental turnover across the Cretaceous/Paleogene transition based on benthic foraminifera.] Revista Española de Paleontología, 23 (1), 15-30. ISSN 0213-6937

\begin{abstract}
The Cretaceous/Paleogene (K/Pg) boundary marks one of the largest mass extinctions in the Fanerozoic, affecting both marine and terrestrial communities. Most scientists accept that the K/Pg boundary extinction was caused, at least in part, by a meteorite impact north of Yucatan peninsula. Such an impact would trigger a series of anomalous deposits, with different characteristics depending on the distance from the impact site; moreover, it triggered a series of dramatic biological, oceanic and climatic changes. Although the impact hypothesis has been broadly accepted, it has originated an intense debate as to the direct or indirect mechanisms that caused the extinctions. Whereas planktic foraminifera and calcareous nannoplancton suffered important mass extinctions, benthic foraminifera do not show any mass extinction across the K/Pg boundary, but temporal changes in the composition of their assemblages. A synthesis of the evolution of benthic foraminiferal assemblages across the $\mathrm{K} / \mathrm{Pg}$ boundary in sections and cores from the Gulf of Mexico and North Atlantic, as well as from more distal areas such as Northern Pacific, South Atlantic and Tethys, is here presented.

Changes in benthic foraminiferal assemblages across the K/Pg boundary have been interpreted as the result of the collapse of the pelagic food web and the scarcity of food supply to the benthos, resulting from the mass extinction of calcareous plankton. However, changes in the composition of benthic assemblages after the K/Pg boundary were geographically variable, and there is no agreement as to the detailed paleoenvironmental interpretation of the assemblages (anoxic or eutrophic conditions in Spanish sections, oligotrophic conditions in Tunisian sections, high and unstable organic flux in N Pacific, etc).

There is evidence that productivity and food transfer to the sea floor recovered much faster than assumed: assemblages from the lower Danian contain short quantitative peaks of opportunistic species that indicate environmental instability, which could be related to the occurrence of blooms of certain primary producers as soon as the light conditions recovered after the impact. This could account for the lack of mass extinctions of benthic foraminifera. Paleoenvironmental changes in the deep sea thus varied geographically, with important differences in the degree and duration of the primary productivity collapse expressed in terms of biomass rather than in terms of diversity. Paleoenvironmental conditions did not recover in the studied sections and cores until, at least, $\sim 300 \mathrm{ky}$ after the K/Pg boundary.
\end{abstract}

Keywords: Benthic foraminifera, Cretaceous, Paleogene, paleoenvironmental changes, impact hypothesis.

\section{RESUMEN}

El límite Cretácico/Paleógeno (K/Pg) marca una de las mayores extinciones en masa del Fanerozoico, tanto en medios marinos como en medios continentales. La mayoría de los científicos acepta que la extinción del límite K/Pg fue al menos en parte originada por el impacto de un meteorito al Norte de la península de Yucatán. El impacto originaría una serie de depósitos anómalos, cuyas características varían en función de la distancia al lugar de impacto; asimismo, provocó una serie de drásticos cambios biológicos, oceánicos y climáticos. Aunque la hipótesis impactista ha sido ampliamente aceptada, se ha generado un intenso debate sobre los mecanismos directos o indirectos mediante los cuales el impacto habría causado las extinciones. Mientras los foraminíferos planctónicos y el nanoplancton calcáreo sufrieron importantes extinciones en masa, los foraminíferos bentónicos 
no experimentaron extinciones masivas en el límite $\mathrm{K} / \mathrm{Pg}$, pero sí muestran cambios temporales en la composición de sus asociaciones. En este trabajo se muestra una síntesis de la evolución de las asociaciones de foraminíferos bentónicos halladas en materiales del tránsito $\mathrm{K} / \mathrm{Pg}$ procedentes de varios cortes y sondeos del área del Golfo de México y Atlántico Noroeste, así como de áreas más distales como el Pacífico Norte, Atlántico Sur y Tethys.

Los cambios experimentados por los foraminíferos bentónicos en el límite $\mathrm{K} / \mathrm{Pg}$ han sido interpretados como resultantes del colapso de la cadena alimenticia pelágica y de la escasez de aporte alimenticio al medio bentónico, como consecuencia de la extinción en masa del plancton calcáreo. No obstante, los cambios en la composición de las asociaciones bentónicas tras el límite $\mathrm{K} / \mathrm{Pg}$ fueron geográficamente variables, y no existe un consenso en cuanto a la interpretación paleoambiental detallada de las asociaciones (condiciones anóxicas o eutróficas en secciones españolas, oligotrofia en perfiles tunecinos, un flujo de nutrientes muy alto e inestable en el Pacífico Norte, etc).

Existen evidencias de que la productividad y la transferencia de alimento al fondo marino se recuperaron más rápido de lo que se asume: las asociaciones del Daniense basal contienen cortos picos cuantitativos de especies oportunistas que indican inestabilidad ambiental, y que podrían estar relacionadas con la proliferación de determinados productores primarios en cuanto se recuperaran las condiciones de luz tras el impacto. Todo ello podría explicar la ausencia de extinción de foraminíferos bentónicos. Así, los cambios paleoambientales en medios profundos variaron geográficamente, con importantes diferencias en el grado y duración del colapso en la productividad primaria expresada en términos de biomasa más que en términos de diversidad. Las condiciones paleoambientales no se recuperaron en los cortes y sondeos estudiados hasta, por lo menos, unos 300 ka tras el límite $\mathrm{K} / \mathrm{Pg}$.

\section{Palabras clave: Foraminíferos bentónicos, Cretácico, Paleógeno, cambios paleoambientales, hipótesis im- pactistas.}

\section{INTRODUCCIÓN}

Durante las últimas décadas, se han estado investigando de forma intensiva cortes y sondeos del Cretácico Superior y Paleógeno inferior distribuidos por todo el mundo, debido a que en el límite Cretácico/Paleógeno (K/Pg) se produjo una de las mayores extinciones en masa de todo el Fanerozoico. Las hipótesis sobre la causa de las extinciones tanto en medios continentales como en medios marinos, sobre el origen de los depósitos característicos del K-Pg y sobre la evolución paleoambiental siguen siendo objeto de debate. La mayoría de los autores aceptan la hipótesis de un impacto meteorítico (Álvarez et al., 1980; Smit \& Hertogen, 1980) en la península de Yucatán (Sureste de México) como la principal causa de las extinciones y de las concentraciones anómalas de iridio, cuarzos de choque y microesférulas en el límite K/Pg (Smit \& Ten Kate, 1982; Hildebrand et al., 1991; Sharpton et al., 1992). Mientras en áreas lejanas al lugar de impacto se observa una capa centimétrica de arcillas con una concentración anómala de iridio, en áreas más próximas (Golfo de México, Caribe y Atlántico Noroeste) se depositó un complejo clástico que ha sido ampliamente aceptado como resultado del impacto, que desestabilizó los márgenes continentales (Bohor, 1996; Smit et al., 1996; Bralower et al., 1998; Soria et al., 2001; Arz et al., 2004). Sin embargo, un reducido grupo de investigadores no apoya la hipótesis impactista, y sugieren que las extinciones no fueron catastróficas sino escalonadas, comenzando en el Maastrichtiense (Keller, 1989 a, b, 2003). Según estos autores, la unidad clástica del K/Pg se depositó como resultado de cambios relativos del nivel del mar o de la tectónica regional (Keller \& Stinnesbeck, 1996; Keller et al., 1997). No obstante, el patrón de extinción gradual y el depósito de los sedimentos del K/Pg a lo largo de un extenso periodo de tiempo resultan de una incorrecta interpretación del registro fósil, y son resultado de discontinuidades, bioturbación, y reelaboración de los fósiles (Signor \& Lipps, 1982; Alegret et al., 2003).

El impacto de un cuerpo extraterrestre en coincidencia con el límite $\mathrm{K} / \mathrm{Pg}$ generó una serie de drásticos cambios biológicos, oceánicos y climáticos (Hsü et al., 1982; Hsü \& McKenzie, 1985; Smit \& Romein, 1985; d'Hondt, 2005) que se han interpretado como causantes de las extinciones. Mientras los foraminíferos planctónicos y el nanoplancton calcáreo sufrieron importantes extinciones en masa (ej., Smit, 1990; Gardin \& Monechi, 1998; Molina et al., 1998; Hollis, 2003) y muestran baja diversidad y abundancia a comienzos del Paleoceno, los foraminíferos bentónicos muestran cambios temporales en la composición de sus asociaciones, extinción de sólo unas pocas especies, y son comunes tras el límite K/Pg (Culver, 2003; Alegret \& Thomas, 2005). Los foraminíferos bentónicos, además de ser unos excelentes marcadores paleobatimétricos, son útiles como marcadores del aporte de nutrientes y de las condiciones de oxigenación en el fondo marino, constituyendo por tanto una importante herramienta para inferir los cambios paleoambientales en el tránsito $\mathrm{K} / \mathrm{Pg}$.

Los cambios temporales en la estructura de las asociaciones de foraminíferos bentónicos de medios profundos han sido interpretados como resultantes del colapso de la cadena alimenticia pelágica y de la escasez de aporte alimenticio al medio bentónico (ej., Kuhnt \& Kaminski, 
1993; Thomas, 1990a, b; Alegret et al., 2001, 2003). No obstante, en algunos cortes el aporte alimenticio aparentemente aumentó tras el límite K/Pg (ej., cortes de Caravaca, Coccioni et al., 1993; y de Agost, en España, Alegret et al., 2003; sondeo DSDP Site 465 en el Pacífico, Alegret \& Thomas, 2005). En algunas de estas localidades (Caravaca, Agost) los sedimentos depositados justo tras el límite K/Pg reflejan condiciones anóxicas (sedimentos laminados), como ocurre en zonas de alta productividad en los océanos actuales. Existen por tanto considerables diferencias regionales en los efectos del evento del $\mathrm{K} / \mathrm{Pg}$ sobre el flujo alimenticio al fondo oceánico.

Resulta difícil comprender cómo los relativamente escasos y reversibles cambios en las asociaciones de foraminíferos bentónicos pudieron ser la respuesta a un colapso de la productividad oceánica, que supuestamente duró cientos de miles de años (Strangelove Ocean; Hsü et al., 1982; Hsü \& Mackenzie, 1985), según se ha deducido de la desaparición del gradiente de los isótopos del carbono bentónico y planctónico (ej. Arthur et al., 1979). Posiblemente, los foraminíferos bentónicos finicretácicos tenían una relación con el medio pelágico menos estrecha que en la actualidad (Thomas et al., 2000), pero deberían de haber sufrido cambios mucho más drásticos si el aporte alimen- ticio hubiera permanecido extremadamente bajo durante cientos de miles de años.

Por otro lado, muchos de los cortes situados en áreas próximas al cráter de impacto están incompletos debido a los procesos de desprendimiento de masas, slumps y deslizamientos submarinos relacionados con la desestabilización de los márgenes continentales ocasionada por el impacto en Chicxulub (Bralower et al., 1998; Klaus et al., 2000; Soria et al., 2001). La comparación de las asociaciones de foraminíferos bentónicos de estos cortes con otros situados en áreas más distales al cráter de impacto proporcionará un registro más completo de la evolución faunística y paleoambiental en el tránsito $\mathrm{K} / \mathrm{Pg}$.

\section{MATERIALES Y MÉTODOS}

Se han estudiado las asociaciones de foraminíferos bentónicos halladas en materiales del tránsito $\mathrm{K} / \mathrm{Pg}$ procedentes de varios cortes y sondeos del área del Golfo de México y Atlántico Noroeste, así como de áreas más distales como el Pacífico Norte, Atlántico Noreste y Sureste, y el Tethys (Fig. 1). Los cortes y sondeos analizados, la síntesis estratigráfica de los sedimentos del tránsito

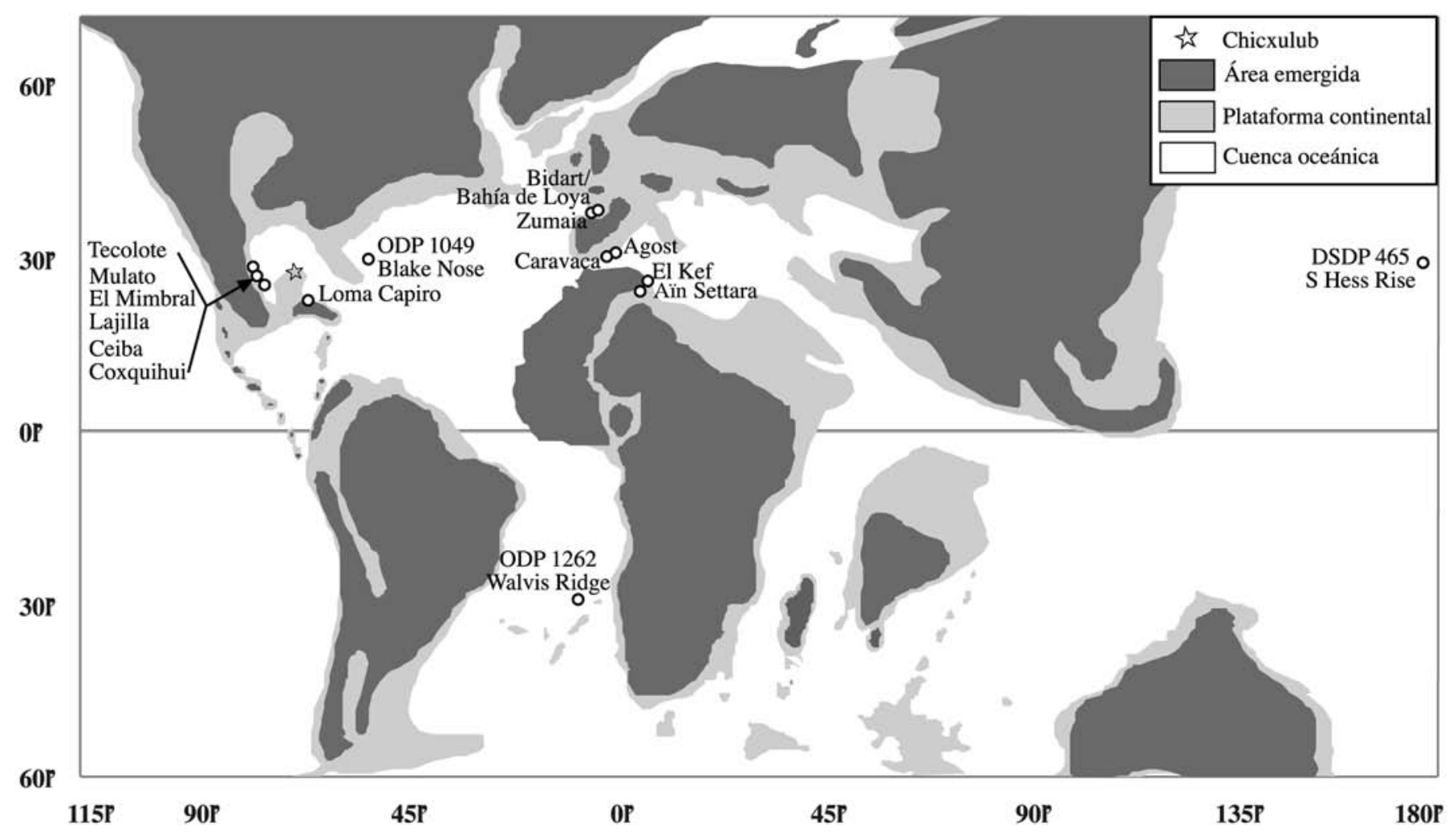

Figura 1. Distribución paleogeográfica de las masas continentales y de las cuencas oceánicas en el límite K/Pg (modificada de Denham \& Scotese, 1987). Se muestra la posición de la estructura de Chicxulub (península de Yucatán, México) y de los cortes y sondeos estudiados.

Paleogeographical distribution of continental masses and oceanic basins at the K/Pg boundary (modified from Denham \& Scotese, 1987). The location of the Chicxulub structure (Yucatán peninsula, México) and the studied sections and cores are shown. 


\begin{tabular}{|c|c|c|c|c|c|c|c|c|c|c|c|c|c|c|c|c|c|c|c|c|c|}
\hline 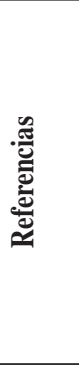 & & 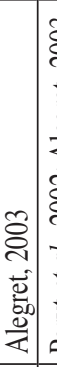 & 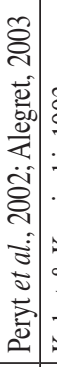 & 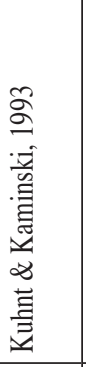 & 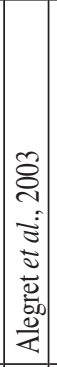 & 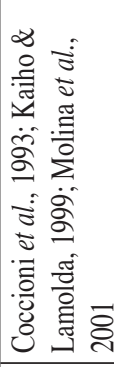 & 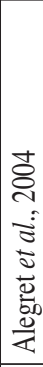 & 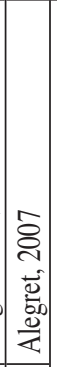 & &  & 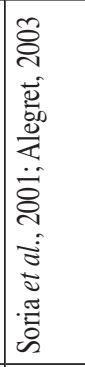 & 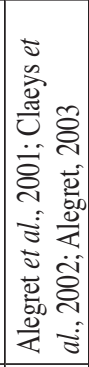 & 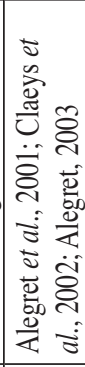 & 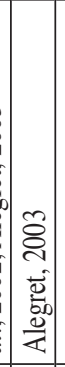 & 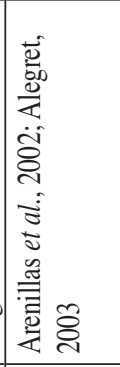 & 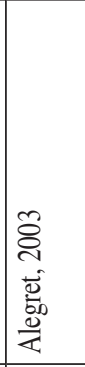 & 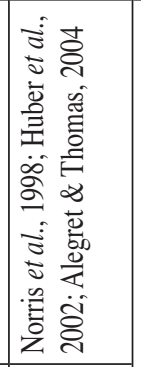 & & 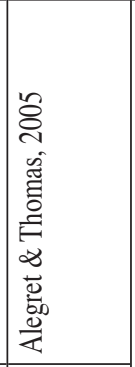 & & 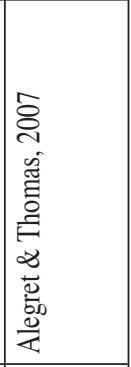 \\
\hline 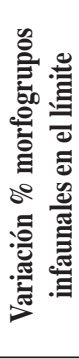 & & 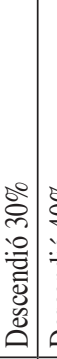 &  & 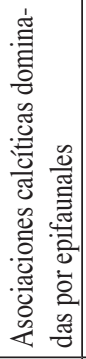 & 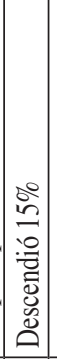 & $\begin{array}{l}\text { 号 } \\
\text { 总 } \\
\text { 丟 }\end{array}$ & 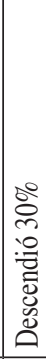 & 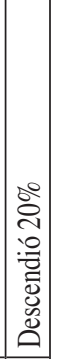 & & 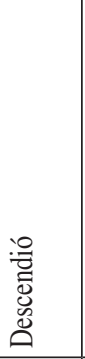 & & 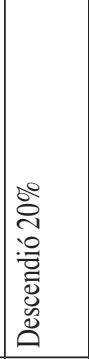 & 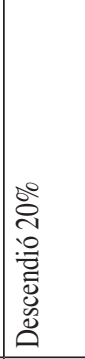 & 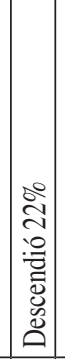 & 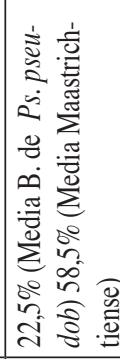 & 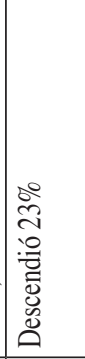 & 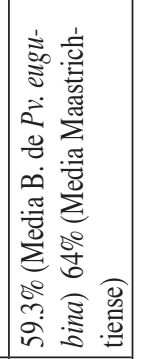 & & 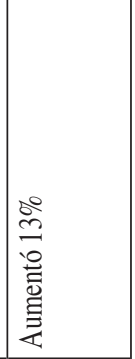 & & 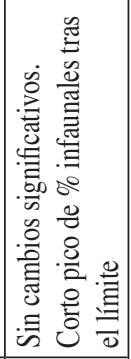 \\
\hline 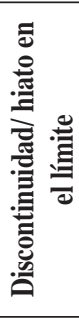 & & $z=$ & \&: & $z^{\circ}$ & $z$ & $z$ & 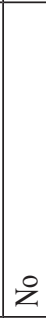 & $z$ & & 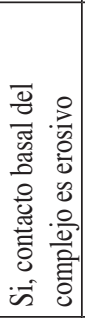 & in & 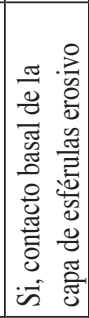 & 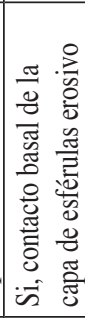 & & 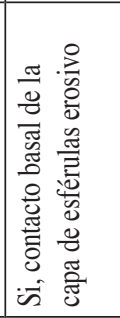 & 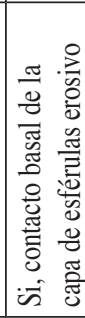 & 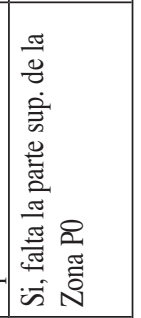 & & 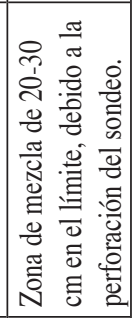 & & \& \\
\hline  & & 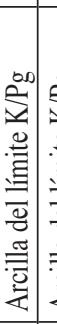 & 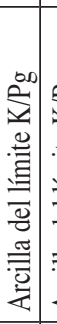 & 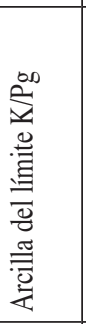 & 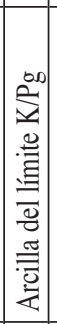 & 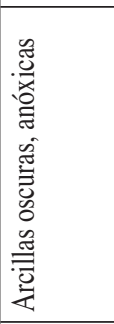 & 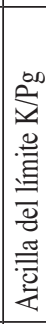 & 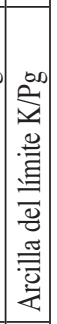 & &  & 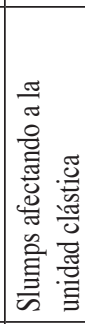 & 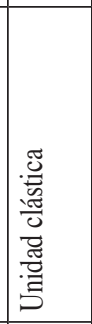 & 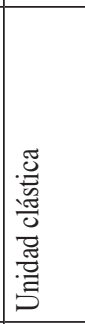 & 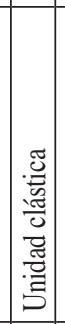 & 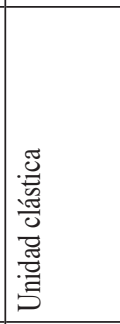 & 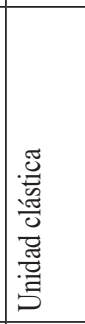 & 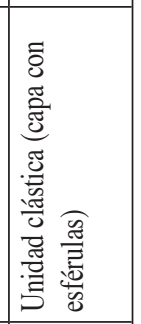 & & 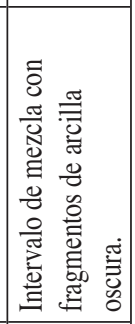 & & 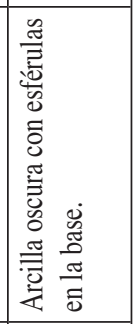 \\
\hline 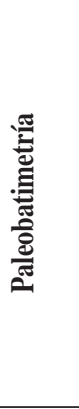 & & 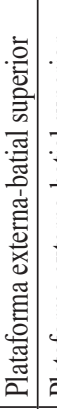 &  & 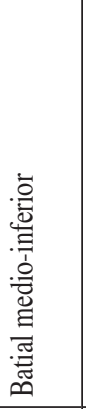 & 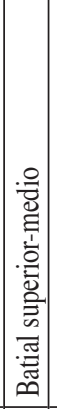 & 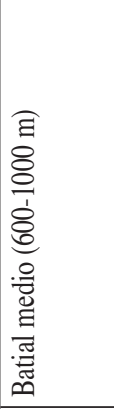 & 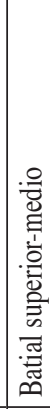 & 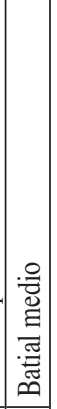 & & 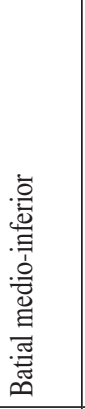 &  & 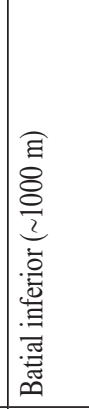 & 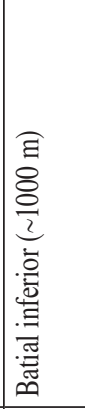 & 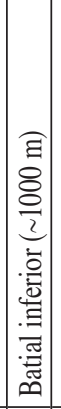 & 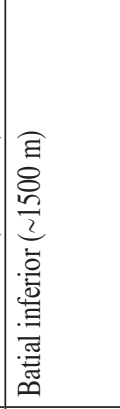 & 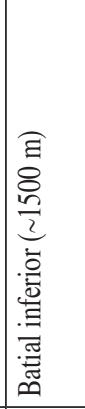 & 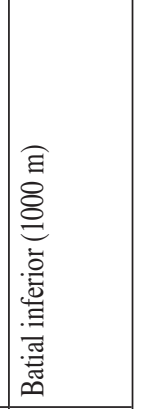 & & 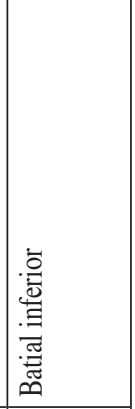 & & 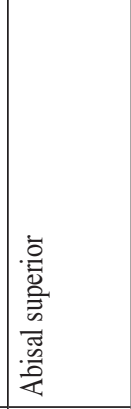 \\
\hline 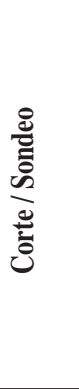 & 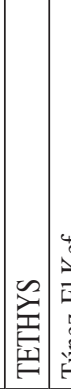 & 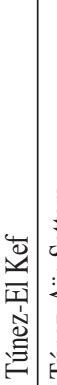 &  & 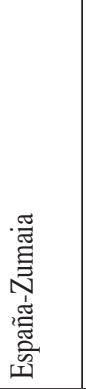 & 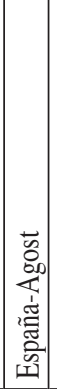 & 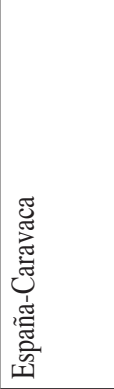 & & 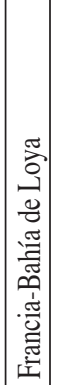 & 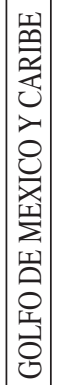 & 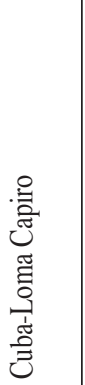 &  & 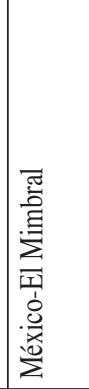 & 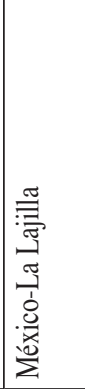 &  & 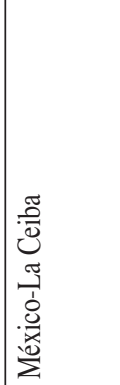 & 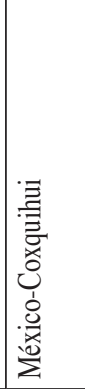 & 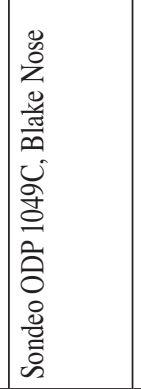 & & 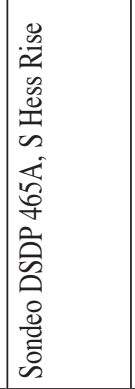 & & 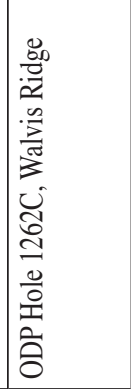 \\
\hline
\end{tabular}

Tabla I. Síntesis estratigráfica de los sedimentos del tránsito K/Pg, paleobatimetrías correspondientes (basadas en el análisis de los foraminíferos bentónicos), y variación en el porcentaje de morfogrupos de foraminíferos bentónicos infaunales en los cortes y sondeos analizados.

Stratigraphic synthesis of K/Pg boundary sediments, paleobathymetry (based on benthic foraminiferal analyses), and changes in the percentages of infaunal benthic foraminiferal morphogroups in the studied sections and cores. 
$\mathrm{K} / \mathrm{Pg}$, y sus paleobatimetrías correspondientes (basadas en el análisis de los foraminíferos bentónicos) se detallan en la Tabla I.

Mientras en áreas lejanas al lugar de impacto, incluido el corte estratotípico de El Kef (Túnez), se observa una capa centimétrica de arcillas con una concentración anómala de iridio (Fig. 2), en áreas más próximas (Golfo de México, Caribe y Atlántico Noroeste) se depositó un complejo clástico cuyo origen se ha relacionado con la desestabilización de los márgenes continentales ocasionada por el impacto meteorítico en Chicxulub (Bralower et al., 1998; Soria et al., 2001). La energía del impacto provocaría enormes olas-tsunami, afectando principalmente a las áreas costeras adyacentes (Golfo de México, Caribe y Atlántico Noroeste; Bourgeois et al., 1988). El resultado sería el depósito de un complejo clástico muy característico con evidencias de impacto, fundamentalmente microtectitas (Smit et al., 1996).

Como consecuencia de los procesos de desprendimiento de masas, slumps y deslizamientos submarinos relacionados con la desestabilización de los márgenes continentales, muchos de los cortes situados en áreas próximas al cráter de impacto están incompletos (Soria et al., 2001; Alegret $\&$ Thomas, 2005). Por este motivo es interesante estudiar cortes y sondeos más alejados del área de impacto, don- de la serie estratigráfica se encuentra más completa; además, en algunas regiones localizadas (ej. Agost y Caravaca en España) se observan arcillas laminadas oscuras con evidencias de anoxia justo en la base del Daniense (Alegret et al., 2003). La comparación de cortes próximos al cráter de impacto con cortes y sondeos situados en áreas más lejanas permitirá obtener un registro más completo de la evolución de los foraminíferos bentónicos y de las condiciones paleoambientales a través del tránsito $\mathrm{K} / \mathrm{Pg}$. Para ello, en el presente trabajo se realiza una síntesis de la evolución de los foraminíferos bentónicos y de las inferencias paleoambientales obtenidas en diversos cortes y sondeos del Golfo de México y Atlántico Noroeste, asî como de áreas más distales como el Pacífico Norte, Atlántico Noreste y Sureste, y el Tethys (Fig. 1). Además, se aportan datos inéditos sobre las asociaciones de foraminíferos bentónicos del corte estratotípico del límite $\mathrm{K} / \mathrm{Pg}$, situado en El Kef (Túnez).

En todos los casos se ha tratado de realizar muestreos de alta resolución. Las muestras fueron disgregadas en agua con $\mathrm{H}_{2} \mathrm{O}_{2}$ y lavadas a través de un tamiz de $63 \mu \mathrm{m}$ para la obtención de los microfósiles. Para realizar los estudios cuantitativos de las asociaciones se separaron unos 300 ejemplares de foraminíferos bentónicos de cada muestra, en la fracción mayor de $63 \mu \mathrm{m}$. Todos los ejemplares fueron montados en celdillas para su conservación e identificación. La clasificación de los foraminíferos a



Figura 2. Distribución y abundancia relativa de las especies de foraminíferos bentónicos más características del tránsito K/Pg en el corte estratotípico de El Kef (Túnez). P.= Plummerita; G. cre.= Güembelitria cretacea; Pv.= Parvularugoglobigerina; Ps. = Parasubbotina. Bioestratigrafía de Molina et al. (2006).

Distribution and relative abundance of the most characteristic benthic foraminiferal species across the K/Pg transition at the stratotype section of El Kef (Tunisia). P.= Plummerita; G. cre.= Güembelitria cretacea; Pv.= Parvularugoglobigerina; Ps.= Parasubbotina. Biostratigraphy by Molina et al. (2006). 
nivel genérico sigue los criterios de Loeblich \& Tappan (1987). Cuando fue posible, los foraminíferos bentónicos se identificaron a nivel de especie. En la figura 2 se muestra la abundancia relativa de las especies de foraminíferos bentónicos más características identificadas en el corte de El Kef; en el apéndice I se ha incluido una lista taxonómica con las referencias originales de las especies representadas en la figura 2. Además, se calculó la abundancia relativa de las especies, el índice de diversidad Fisher- $\alpha$ y el de heterogeneidad H(S) Shannon-Weaver (Murray, 1991). En la figura 3 se muestran las variaciones de estos índices en el corte estratotípico del límite $\mathrm{K} / \mathrm{Pg}$ (El Kef), y en las figuras 4 y 5 se muestran fotografías de los taxones más representativos en dicho corte.

Los foraminíferos bentónicos son buenos indicadores en reconstrucciones paleobatimétricas. La comparación entre asociaciones fósiles y actuales, la presencia y abundancia de especies relacionadas con la profundidad, y sus límites superiores (ej., Van Morkhoven et al., 1986; Alegret et al., 2003) han permitido inferir la paleobatimetría. Todos los ejemplares de foraminíferos bentónicos han sido asignados a morfogrupos siguiendo los criterios de Corliss (1985), Jones \& Charnock (1985) y Corliss \& Chen (1988). La comparación de asociaciones fósiles y actuales, y el análisis morfotípico, permiten inferir los microhábitats y los parámetros ambientales como el aporte de nutrientes al fondo marino o la oxigenación en las aguas del fondo (ej., Bernhard, 1986; Jorissen et al., 1995, 2007; Fontanier et al., 2002). No obstante, hay que tener cierta cautela con la interpretación de estas comparaciones, porque no sabemos hasta qué punto las asociaciones del Cretácico y Paleógeno inferior eran análogas a las actuales (ej., Thomas et al., 2000), e incluso en las actuales no se conoce con certeza el microhábitat de muchas especies de medios profundos (ej., Buzas et al., 1993).

\section{RESULTADOS Y DISCUSIÓN}

Las asociaciones de foraminíferos bentónicos del Maastrichtiense superior son diversas en todos los cortes y sondeos estudiados, incluyendo el corte estratotípico (Fig. 3), están dominadas por morfogrupos infaunales (o infaunales y epifaunales), e indican condiciones ambientales estables y mesotróficas. La abundancia relativa de los morfogrupos infaunales se incrementa hacia el Maastrichtiense terminal en los perfiles mexicanos, sugiriendo un mayor flujo en el aporte de nutrientes al fondo marino a finales del Cretácico. Sin embargo, la abundancia de bulimínidos en Blake Nose (50\%) y Hess Rise (hasta 40\%) indica que la productividad en el Atlántico Noroeste y Pacífico Norte fue mayor que en el Golfo de México. Los bulimínidos toleran bajas concentraciones de oxígeno, aunque su abundancia está relacionada fundamentalmente con un abundante flujo alimenticio y con fluctuaciones anuales o estacionales en el aporte al fondo marino (ej., Fontanier et al., 2002; Jorissen et al., 2007). El alto porcentaje de bulimínidos y de

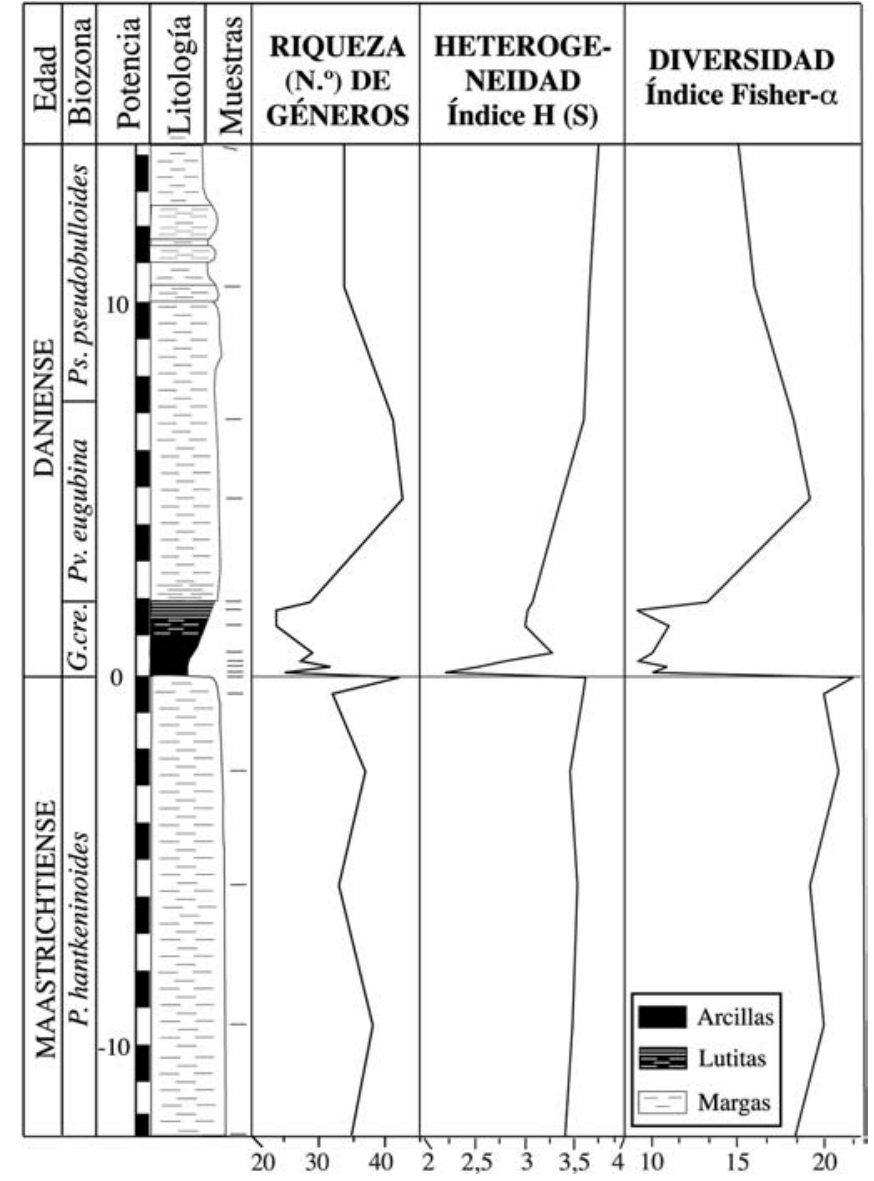

Figura 3. Índices de diversidad, heterogeneidad y riqueza genérica de foraminíferos bentónicos en el corte estratotípico de El Kef (Túnez). P.= Plummerita; G. cre.= Güembelitria cretacea; Pv. = Parvularugoglobigerina; Ps.= Parasubbotina. Bioestratigrafía de Molina et al. (2006).

Diversity, heterogenety and genus richness indices of benthic foraminifera at the stratotype section of $\mathrm{El}$ Kef (Tunisia). P.= Plummerita; G. cre.= Güembelitria cretacea; Pv. $=$ Parvularugoglobigerina; Ps. $=$ Parasubbotina. Biostratigraphy by Molina et al. (2006).

morfogrupos infaunales en Blake Nose, y la escasez de especies típicas de medios batiales oligotróficos, como Stensioeina beccariiformis (White, 1928), Cibicidoides hyphalus (Fisher, 1969), Nuttallides truempyi (Nuttall, 1930) y Gyroidinoides globosus (Hagenow, 1842), sugieren que este sondeo se situaba en un área de corrientes de surgencia (Alegret \& Thomas, 2004). El porcentaje de taxones aglutinados es mayor en los cortes mexicanos (30-62\%) que en el resto de cortes y sondeos, e indica un importante flujo de detritos hacia la cuenca en esta zona.

Los foraminíferos hallados en la capa de esférulas en Blake Nose, y en las unidades clásticas de los perfiles mexicanos y cubanos varían mucho en el tipo de conservación, y las asociaciones consisten en una mezcla de espe- 
cies típicas de medios neríticos y otras de medios batiales (Alegret et al., 2001, 2005). Estas asociaciones debieron ser el resultado del arrastre de sedimentos desde la plataforma y su transporte a lo largo del talud, como consecuencia del impacto del límite $\mathrm{K} / \mathrm{Pg}$. La presencia de componentes neríticos, alóctonos, apoya la hipótesis de que el depósito de estas unidades en la parte media e inferior del talud fue originado por un evento único y geológicamente instantáneo relacionado con extensos desprendimientos de masas y slumps (Alegret et al., 2001, 2005; Soria et al., 2001; Arenillas et al., 2002).

Mientras los foraminíferos planctónicos y el nanoplancton calcáreo fueron afectados por una extinción en masa catastrófica en el límite K/Pg (ej. Arz et al., 2001; Arenillas et al., 2002), las tasas de extinción de los foraminíferos bentónicos no superan el $10 \%$ en ninguno de los cortes estudiados. Más que una extinción en masa, los foraminíferos bentónicos muestran una reorganización de la estructura de sus comunidades, incluyendo la desaparición temporal de taxones Lázaro y un drástico descenso en la diversidad, heterogeneidad y riqueza de géneros (Figs. 2 y 3). En coincidencia con el límite K/Pg se registra un drástico descenso en el porcentaje de los morfogrupos infaunales (25-45\%) en los cortes mexicanos y españoles, asî como en el sondeo de Blake Nose (Atlántico Noroeste). Este descenso, y el descenso en el porcentaje de bulimínidos, sugiere una drástica disminución en el aporte alimenticio al fondo marino, relacionado con la extinción del plancton calcáreo en el límite K/Pg (d'Hondt et al., 1998; Alegret et al., 2001). Una situación similar se observa en los perfiles tunecinos; en el corte de El Kef, el porcentaje de los morfogrupos infaunales descendió un 30\% en coincidencia con el límite K/Pg (Fig. 3). No obstante, esta disminución en el aporte alimenticio fue menos drástica en Blake Nose (Atlántico Noroeste) y en Hess Rise (Pacífico Norte), donde las asociaciones no reflejan condiciones oligotróficas severas, sino un descenso moderado en el aporte alimenticio seguido de una rápida recuperación.

Al contrario de lo que ocurre en la mayoría de los cortes (incluyendo el corte estratotípico de El Kef, Fig. 3) y sondeos, no se observan cambios en la composición de los morfogrupos en el límite K/Pg en el sondeo 1262 de Walvis Ridge (Atlántico Sureste), donde el aumento de los morfotipos infaunales "redondeados planispiralados" y "esféricos" como Pullenia Parker \& Jones, 1862, Globulina d'Orbigny, 1839 o Quadrimorphina Finlay, 1939 compensa la pérdida de morfotipos infaunales "alargados y cilíndricos" (Alegret \& Thomas, 2007). En este sondeo, el drástico descenso en la tasa de acumulación de foraminíferos bentónicos en el límite $\mathrm{K} / \mathrm{Pg}$, y el descenso en el porcentaje de bulimínidos, sugieren una disminución en el aporte alimenticio, pero esto se contradice con la ausencia de cambios en el porcentaje de los morfogrupos infaunales. La abundancia de taxones oportunistas, y las fluctuaciones en la diversidad, heterogeneidad y en la tasa de acumulación de foraminíferos bentónicos, sugieren que tras el límite K/Pg hubo un periodo de fuertes fluctuaciones en el aporte alimenticio.

Aunque algunos autores (Kyte et al., 1980; Kajiwara \& Kaiho, 1992) han sugerido que el fondo del océano pudo ser anóxico tras el límite $\mathrm{K} / \mathrm{Pg}$, únicamente existen evidencias de anoxia local o regional (ej., en la Fish Clay de Dinamarca, Coccioni \& Galeotti, 1998; o en varias localidades del Tethys, Martínez-Ruiz et al., 1992; Coccioni et al., 1993; Kaiho et al., 1999). No obstante, la anoxia o disoxia no se extendió a lo largo de todo el Tethys (Peryt et al., 2002). No se han hallado evidencias de baja oxigenación en los cortes tunecinos (incluyendo el corte estratotípico de El Kef), donde la abundancia de especies con grandes poros en sus conchas como Cibicidoides pseudoacutus (Nakkady, 1950) (Fig. 4d-e) es típica de medios bien oxigenados. Tampoco se observan condiciones de escasa oxigenación en los perfiles mexicanos ni en Blake Nose, aunque un corto episodio de anoxia podría haber desaparecido de su registro sedimentario como resultado de los desprendimientos de masas registrados en esta zona (ej., Klaus et al., 2000; Alegret \& Thomas, 2005).

$\mathrm{La}$ anoxia ha sido relacionada por algunos autores con un exceso de biomasa, resultante de los organismos que murieron en las extinciones (Kyte et al., 1980; Coccioni et al., 1993; Coccioni \& Galeotti, 1998), pero Alegret et al. (2003) discutieron que esta hipótesis es cuantitativamente improbable, dado que la biomasa oceánica en la actualidad supone sólo una pequeña fracción de la biomasa total, y la oxidación de incluso toda la biomasa oceánica no llegaría a consumir suficiente oxígeno como para mantener los oceános anóxicos durante miles de años. Una explicación para la anoxia podría ser la oxidación del metano de los gases hidratados liberados por la desestabilización de los márgenes continentales tras el impacto del $\mathrm{K} / \mathrm{Pg}$ (Alegret et al., 2003).

Al igual que en el corte estratotípico de El Kef, en otros cortes y sondeos del Tethys (ej. Caravaca, Agost, Aïn Settara), del Atlántico Sureste (Walvis Ridge), del Pacífico Norte (Hess Rise), Atlántico Noroeste (Blake Nose) y Atlántico Noreste (Bidart, Bahía de Loya), las asociaciones del Daniense basal contienen cortos picos cuantitativos $(<$ Zona $\mathrm{P} \alpha)$ de especies oportunistas que indican inestabilidad ambiental, concretamente en el aporte alimenticio (Alegret \& Thomas, 2005, 2007; Alegret, 2007). Aun así, queda abierta la pregunta de qué cambios paleoambientales provocaron la aparición de estos picos de especies oportunistas a nivel global. En numerosas localidades, el aporte alimenticio al medio bentónico (según los índices de foraminíferos bentónicos) disminuyó durante varios miles de años tras el límite K/Pg, pero en Hess Rise el aporte alimenticio parece haber aumentado; en algunos cortes del Tethys (ej., Caravaca, Agost) el desarrollo de condiciones de baja oxigenación refleja un incremento aún mayor en el flujo de carbono orgánico. La ausencia de extinciones de 


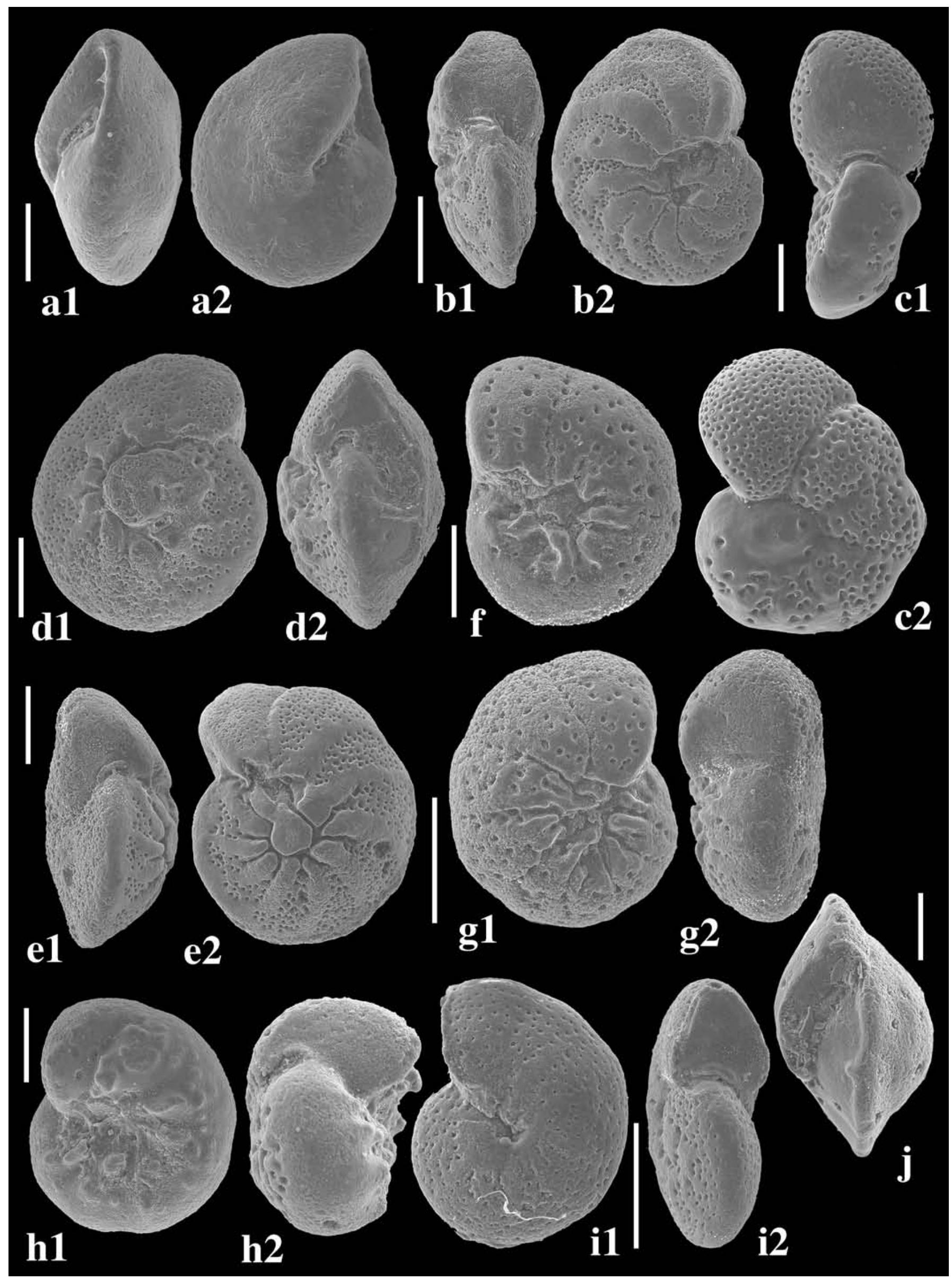


foraminíferos bentónicos en medios profundos indica que no hubo anoxia global tras el límite $\mathrm{K} / \mathrm{Pg}$, y que no sólo el aporte alimenticio se recuperó más rápido de lo sugerido (modelo Strangelove Ocean; Hsü \& McKenzie, 1985), sino que también su transporte al fondo marino se recuperó más rápido que los resultados obtenidos en el modelo del "Océano vivo" propuesto por Coxall et al. (2006).

La biomasa de los productores primarios en los océanos y el flujo de materia orgánica al fondo debieron de recuperarse más rápido que la diversidad de la biota planctónica, a través de la proliferación masiva de taxones oportunistas restringidos a regiones determinadas, tal y como muestra la distribución geográficamente limitada de arcillas negras laminadas. Estos cortos picos de fitoplancton generaron un aporte de alimento al fondo oceánico muy variable en calidad y cantidad, provocando estrés medioambiental en las asociaciones bentónicas. Dicho estrés pudo deberse a un alto aporte alimenticio por parte de productores primarios que serían difíciles de digerir por los foraminíferos bentónicos, como diversas especies de dinoflagelados (Alegret et al., 2003; Alegret \& Thomas, 2004). Así, los cambios paleoambientales en medios profundos variaron geográficamente, con importantes diferencias en el grado y duración del colapso en la productividad primaria expresada en términos de biomasa más que en términos de diversidad. Las condiciones paleoambientales no se recuperaron en los cortes y sondeos estudiados hasta, por lo menos, la segunda mitad de la Biozona de Parasubbotina pseudobulloides (unos 300.000 años tras el límite K/Pg).

El registro de los isótopos del carbono en conchas de foraminíferos bentónicos y en la fracción carbonatada fina reflejan un rápido colapso de los valores superficiales hasta igualar los valores de las aguas profundas en el límite $\mathrm{K} / \mathrm{Pg}$ debido a un descenso en los valores del sedimento y un incremento en los valores bentónicos. Este registro concuerda con la señal esperada durante un corto colapso de la productividad primaria en el límite $\mathrm{K} / \mathrm{Pg}$. Sin embargo, en algunos sondeos como el Sondeo 1262 de Walvis Ridge (Atlántico Sureste), tras el colapso del límite $\mathrm{K} / \mathrm{Pg}$ se observa un gradiente inverso, con valores muy negativos en los isótopos del sedimento, que muestran un pico unos 200.000 años tras el límite $\mathrm{K} / \mathrm{Pg}$ (Thomas et al., 2007). Una posible explicación del gradiente inverso en los isótopos del carbono sería la presencia de corrientes de surgencia locales o regionales, que en los océanos actuales confieren a los foraminíferos planctónicos valores de los isótopos de carbono más negativos que los que presentan los foraminíferos bentónicos (Smart \& Thomas, 2006). En Walvis Ridge, la alta tasa de acumulación de foraminíferos bentónicos tras el límite $\mathrm{K} / \mathrm{Pg}$ indica un elevado aporte alimenticio al fondo oceánico. Sin embargo, en las mismas muestras se registran valores mínimos de $\delta^{13} \mathrm{C}$ en el sedimento y bajos íncides de diversidad, lo que sugiere que se trataba además de un medio de gran estrés y amplias fluctuaciones ambientales con alteraciones en la estratificación oceánica.

Si la productividad y la transferencia de alimento al fondo marino se recuperaron más rápido de lo asumido hasta el momento, se podría explicar la ausencia de una extinción masiva en los foraminíferos bentónicos. En ese caso, no obstante, se ha de encontrar una explicación para la persistente ausencia de los gradientes de los isótopos de carbono bentónicos-planctónicos. Son necesarios más análisis del $\delta^{13} \mathrm{C}$ en el bentos y en el plancton para explicar por qué estos valores confluyen, e incluso los valores superficiales se llegan a hacer más ligeros que los bentónicos (gradiente inverso) en numerosas localidades, lo que supone la adición de isótopos ligeros del carbono al sistema océano-atmósfera durante largos periodos de tiempo ( $10^{5}$ años). Para concluir, no existe actualmente una explicación de los registros de los isótopos del carbono a través del límite $\mathrm{K} / \mathrm{Pg}$. Es necesario realizar análisis de mayor resolución para documentar si el gradiente negativo se produjo a nivel global (reflejando por tanto el ciclo de carbono global) o a nivel local o regional (reflejando la productividad o el transporte de materia orgánica), o si fue aleatorio (reflejando efectos de la diagénesis). El estudio detallado de las asociaciones de foraminíferos bentónicos puede aportar información sobre los cambios en los medios oceánicos y en el ciclo del carbono oceánico durante el límite $\mathrm{K} / \mathrm{Pg}$.

Figura 4. Fotografías de microscopio electrónico de barrido de los foraminíferos bentónicos más característicos hallados en el corte estratotípico de El Kef (Túnez). a1-2, Alabamina wilcoxensis Toulmin, 1941 (muestra Kef 12,7). b1-2, Anomalinoides susanaensis (Browning in Mallory, 1959) (muestra Kef 21,95). c1-2, Anomalinoides rubiginosus (Cushman, 1926) (muestra Kef 21,95). d1-2, Cibicidoides pseudoacutus (Nakkady, 1950) (muestra Kef 12,7). e1-2, Cibicidoides pseudoacutus (Nakkady, 1950) (muestra Kef 21,95). f, g1-2, Stensioeina beccariiformis (White, 1928) (muestra Kef 21,95). h1-2, Sliteria varsoviensis Gawor-Biedowa, 1992 (muestra Kef 3). i1-2, Anomalinoides ammonoides (Reuss, 1844) (muestra Kef 21,95). j, Oridorsalis plummerae (Cushman, 1948) (muestra Kef 21,95). Las escalas equivalen a 100 micras.

Scanning electron-microscope photographs of the most characteristic benthic foraminifera from the stratotype section of El Kef(Tunisia). a1-2, Alabamina wilcoxensis Toulmin, 1941 (sample Kef 12,7). b1-2, Anomalinoides susanaensis (Browning in Mallory, 1959) (sample Kef 21,95). c1-2, Anomalinoides rubiginosus (Cushman, 1926) (sample Kef 21,95). d1-2, Cibicidoides pseudoacutus (Nakkady, 1950) (sample Kef 12,7). e1-2, Cibicidoides pseudoacutus (Nakkady, 1950) (sample Kef 21,95). f, g1-2, Stensioeina beccariiformis (White, 1928) (sample Kef 21,95). h1-2, Sliteria varsoviensis Gawor-Biedowa, 1992 (sample Kef 3). il-2, Anomalinoides ammonoides (Reuss, 1844) (sample Kef 21,95). j, Oridorsalis plummerae (Cushman, 1948) (sample Kef 21,95). Scale bars: 100 microns. 


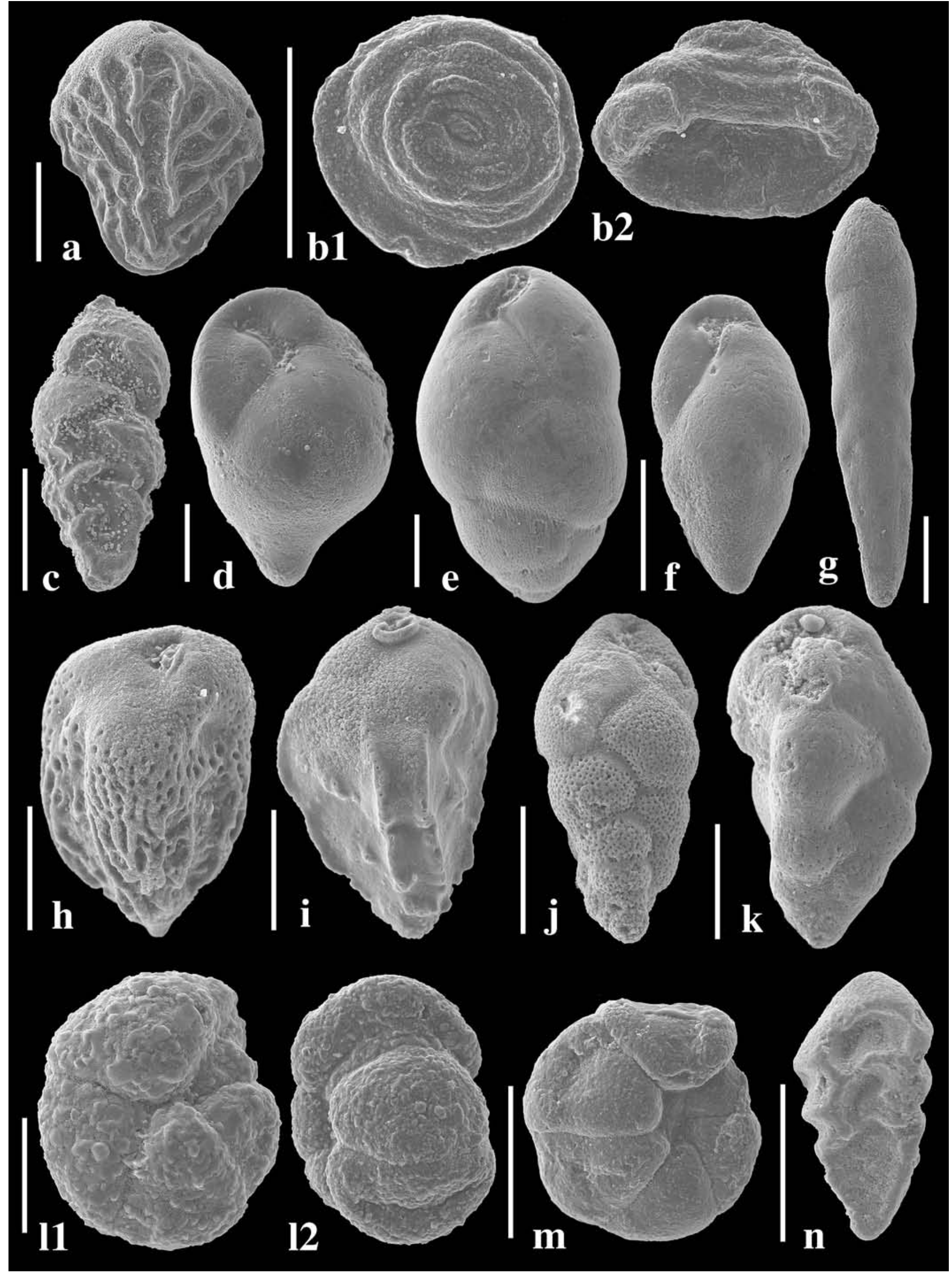




\section{CONCLUSIONES}

Mientras los foraminíferos planctónicos y el nanoplancton calcáreo sufrieron importantes extinciones en masa durante el límite $\mathrm{K} / \mathrm{Pg}$, los foraminíferos bentónicos no experimentaron extinciones significativas, pero sí muestran cambios temporales en la composición de sus asociaciones. Los cambios experimentados por los foraminíferos bentónicos en el límite K/Pg han sido interpretados como resultantes del colapso de la cadena alimenticia pelágica y de la escasez de aporte alimenticio al medio bentónico, como consecuencia de la extinción en masa del plancton calcáreo. Sin embargo, los cambios en la composición de las asociaciones bentónicas tras el límite K/Pg fueron geográficamente variables, y no existe un consenso en cuanto a la interpretación paleoambiental detallada de las asociaciones.

Existen evidencias de que la productividad y la transferencia de alimento al fondo marino se recuperaron más rápido de lo que se asume, a través de la proliferación de determinadas especies de fitoplancton limitadas a regiones determinadas, que generaron un aporte de alimento al fondo oceánico muy variable en calidad y cantidad, provocando estrés medioambiental en las asociaciones bentónicas. Dicho estrés pudo deberse a un elevado aporte alimenticio por parte de productores primarios que serían difíciles de digerir por los foraminíferos bentónicos, como diversas especies de dinoflagelados. Como resultado, las asociaciones del Daniense basal contienen picos cuantitativos de especies de foraminíferos bentónicos oportunistas que indican inestabilidad ambiental.

El desarrollo de un gradiente inverso en los isótopos del carbono bentónicos-planctónicos en algunos sondeos, como en Walvis Ridge, podría deberse a la presencia de corrientes de surgencia locales o regionales. El gradiente inverso desapareció gradualmente, y las condiciones paleoambientales no se recuperaron en los cortes y sondeos estudiados hasta, por lo menos, la segunda mitad de la Biozona de Parasubbotina pseudobulloides ( $300 \mathrm{ka}$ tras el límite $\mathrm{K} / \mathrm{Pg})$.

\section{AGRADECIMIENTOS}

Agradezco las acertadas sugerencias y la labor de edición realizadas por los revisores Alejandro Cearreta y Juan Usera, y por el editor de la revista Rodolfo Gozalo. Asimismo agradezco la inestimable ayuda de mis colegas Ignacio Arenillas, José Antonio Arz y Eustoquio Molina, quienes proporcionaron el marco bioestratigáfico y colaboraron en la recogida de algunas de las muestras. Estre trabajo ha sido financiado por el Ministerio de Ciencia y Tecnología (contrato Ramón y Cajal concedido a la autora, y proyecto Consolider CGL 2007-63724).

\section{REFERENCIAS}

Alegret, L. 2003. Sedimentología y Micropaleontología (foraminíferos bentónicos) del tránsito Cretácico-Terciario: correlación entre las áreas del Golfo de México y del Tethys. Tesis Doctoral. Bell Howell Ed., Madrid, $476 \mathrm{pp}$.

Alegret, L. 2007. Recovery of the deep-sea floor after the Cretaceous/Paleogene boundary event: the benthic foraminiferal record in the Basque-Cantabrian basin and in SE Spain. Palaeogeography, Palaeoclimatology, Palaeoecology, 255, 181-194.

Alegret, L. \& Thomas, E. 2001. Upper Cretaceous and lower Paleogene benthic Foraminifera from northeastern Mexico. Micropaleontology, 47, 269-316.

Alegret, L. \& Thomas, E. 2004. Benthic foraminifera and environmental turnover across the Cretaceous/Paleogene boundary at Blake Nose (ODP Hole 1049C, Northwestern Atlantic). Palaeogeography, Palaeoclimatology, Palaeoecology, 208, 59-83.

Alegret, L. \& Thomas, E. 2005. Paleoenvironments across the Cretaceous/Tertiary boundary in the central North Pacific (DSDP Site 465), the Gulf of Mexico and the Tethys: the benthic foraminiferal record. Palaeogeography, Palaeoclimatology, Palaeoecology, 224, 53-82.

Alegret, L. \& Thomas, E. 2007. Deep-Sea environments across the Cretaceous/Paleogene boundary in the eastern

Figura 5. Fotografías de microscopio electrónico de barrido de los foraminíferos bentónicos más característicos hallados en el corte estratotípico de El Kef (Túnez). a, Bolivinoides draco (Marsson, 1878) (muestra Kef 11,30). b1-2, Arenoturrispirillina tunisiana Peryt et al., 2004 (muestra Kef 12,7). c, Eouvigerina subsculptura McNeil \& Caldwell, 1981 (muestra Kef 6,60). d, Sitella cushmani (Sandidge, 1932) (muestra Kef 0). e, Praeglobobulimina quadrata (Plummer, 1926) (muestra Kef 12,10). f, Sitella fabilis (Cushman \& Parker, 1936) (muestra Kef 9,5). g, Coryphostoma incrassata (Reuss, 1851) (muestra Kef 0). h, Bulimina strobila Marie, 1941 (muestra Kef 9,5). i, Pseudouvigerina plummerae Cushman, 1927 (muestra Kef 9,5). j, Pyramidina prolixa (Cushman \& Parker, 1935) (muestra Kef 9,5). k, Pyramidina cf. prolixa (muestra Kef 9,5). 11-2, m, Haplophragmoides sp. (muestra Kef 12,7). n, Tappanina selmensis (Cushman, 1933) (muestra Kef 16,50). Las escalas equivalen a 100 micras.

Scanning electron-microscope photographs of the most characteristic benthic foraminifera from the stratotype section of El Kef (Tunisia). a, Bolivinoides draco (Marsson, 1878) (sample Kef 11,30). b1-2, Arenoturrispirillina tunisiana Peryt et al., 2004 (sample Kef 12,7). c, Eouvigerina subsculptura McNeil \& Caldwell, 1981 (sample Kef 6,60). d, Sitella cushmani (Sandidge, 1932) (sample Kef 0). e, Praeglobobulimina quadrata (Plummer, 1926) (sample Kef 12,10). f, Sitella fabilis (Cushman \& Parker, 1936) (sample Kef 9,5). g, Coryphostoma incrassata (Reuss, 1851) (sample Kef 0). h, Bulimina strobila Marie, 1941 (sample Kef 9,5). i, Pseudouvigerina plummerae Cushman, 1927 (sample Kef 9,5). j, Pyramidina prolixa (Cushman \& Parker, 1935) (sample Kef 9,5). $\boldsymbol{k}$, Pyramidina $c f$. prolixa (sample Kef 9,5). l1-2, $\boldsymbol{m}$, Haplophragmoides $s p$. (sample Kef 12,7). n, Tappanina selmensis (Cushman, 1933) (sample Kef 16,50). Scale bars: 100 microns. 
South Atlantic Ocean (ODP Leg 208, Walvis Ridge). Marine Micropaleontology, 64, 1-17.

Alegret, L., Molina, E. \& Thomas, E. 2001. Benthic foraminifera at the Cretaceous/Tertiary boundary around the Gulf of Mexico. Geology, 29, 891-894.

Alegret, L., Molina, E. \& Thomas, E., 2003. Benthic foraminiferal faunal turnover across the Cretaceous/Tertiary Boundary at Agost (Southeastern Spain). Marine Micropaleontology, 48, 251-279.

Alegret, L., Kaminski, M. A. \& Molina, E. 2004. Paleoenvironmental recovery after the Cretaceous/Tertiary boundary crisis: evidence from the marine Bidart section (SW France). Palaios, 19, 574-586.

Alegret, L., Arenillas, I., Arz, J. A., Diaz, C., Grajales-Nishimura, J. M., Mélendez, A., Molina, E., Rojas, R. \& Soria, A. R. 2005. Cretaceous-Paleogene boundary deposits at Loma Capiro, Cuba: evidence for the Chicxulub impact. Geology, 33, 721-724.

Alth, A. 1850. Geognostisch-paläontologische Beschreibung der nächsten Umgebung von Lemberg. Haidinger's naturwisschen Abhandlungen, Viena, 3, 171-284.

Álvarez, L. W., Álvarez, W., Asaro, F. \& Michel, H. V. 1980. Extraterrestrial cause for the Cretaceous-Tertiary extinction. Science, 208, 1195-1108.

Arenillas, I., Alegret, L., Arz, J. A., Liesa, C., Meléndez, A., Molina, E., Soria, A. R., Cedillo, E., Grajales, J. M. \& Rosales, C. 2002. Cretaceous-Tertiary boundary planktic foraminiferal mass extinction and biochronology at $\mathrm{La}$ Ceiba and Bochil, Mexico, and El Kef, Tunisia. In: Catastrophic events and mass extinctions: impacts and beyond (eds. C. Koeberl \& K. G. MacLeod). Geological Society of America Special Paper, 356, 253-264.

Arthur, M. A., Scholle, P. \& Hasson, P. 1979. Stable isotopes of oxygen and carbon in carbonates from Sites 398 and 116 of the Deep Sea Drilling Project. Initial Reports DSDP , 47, 477-492.

Arz, J. A., Alegret, L., Arenillas, I., Liesa, C., Molina, E. \& Soria, A. R. 2001. Extinción de foraminíferos en el límite Cretácico/Terciario de Coxquihui (México) y su relación con las evidencias de impacto. Revista Española de Micropaleontología, 33, 221-236.

Arz, J. A., Alegret, L. \& Arenillas, I. 2004. Foraminiferal biostratigraphy and paleoenvironmental reconstruction at Yaxcopoil-1 drill hole (Chicxulub crater, Yucatan Peninsula). Meteoritics \& Planetary Science, 39, 1099-1111.

Bernhard, J. M. 1986. Characteristic assemblages and morphologies of benthic foraminifera from anoxic, organicrich deposits: Jurassic through Holocene. Journal of Foraminiferal Research, 16, 207-215.

Bohor, B. F. 1996. A sediment gravity flow hypothesis for siliciclastic units at the K/T boundary, northeastern Mexico. Geological Society of America Special Paper, 307, 183-196.

Bourgeois J., Hansen T. A., Wiberg P. L. \& Kauffman E. G. 1988. A tsunami deposit at the Cretaceous-Tertiary boundary in Texas. Science, 241, 567-570.

Bralower, T. J., Paull, C. K. \& Leckie, R. M. 1998. The Cretaceous/Tertiary boundary cocktail: Chicxulub impact triggers marine collapse and extensive sediment gravity flows. Geology, 26, 331-334.

Brotzen, F. 1940. Flintrännans och Trindelrännans geologi (Öresund). Sveriges Geologiska Undersökning, C435, 34, 3-33

Brotzen, F. 1948. The Swedish Paleocene and its foraminiferal fauna. Sveriges Geologiska Undersökning, C493, 42, 1-140.

Buzas, M. A., Culver, S. J. \& Jorissen, F. J. 1993. A statistical evaluation of the microhabitats of living (stained) infaunal benthic foraminifera. Marine Micropaleontology, 29, 73-76.

Claeys, Ph., Kiessling, W. \& Alvarez, W. 2002. Distribution of Chicxulub ejecta at the Cretaceous-Tertiary boundary. In: Catastrophic Events and Mass Extinctions: Impacts and Beyond (eds. C. Koeberl \& K. G. MacLeod). Geological Society of America Special Paper, 356, 55-68.

Coccioni, R. \& Galeotti, S. 1998. What happened to small benthic foraminifera at the Cretaceous/ Tertiary boundary? Bulletin de la Société Géologique de France, 169, 271-279.

Coccioni, R., Fabbrucci, L. \& Galeotti, S. 1993. Terminal Cretaceous deep-water benthic foraminiferal decimation, survivorship and recovery at Caravaca (SE Spain). $P a$ laeopelagos, 3, 3-24.

Corliss, B. H. 1985. Microhabitats of benthic foraminifera within deep-sea sediments. Nature, 314, 435-438.

Corliss, B. H. \& Chen, C. 1988. Morphotype patterns of Norwegian Sea deep-sea benthic foraminifera and ecological implications. Geology, 16, 716-719.

Coxall, H. K., D'Hondt, S. \& Zachos, J. C. 2006. Pelagic evolution and environmental recovery after the CretaceousPaleogene mass extinction. Geology, 34, 297-300.

Cushman, J. A. 1926. The foraminifera of the Velasco Shale of the Tampico embayment. American Association of Petroleum Geologists Bulletin, 10, 581-612.

Cushman, J. A. 1927. New and interesting foraminifera from Mexico and Texas. Contributions of the Cushman Laboratory for Foraminiferal Research, 3, 111-117.

Cushman, J. A. 1933. Foraminifera: their classification and economic use. Cushman Laboratory for Foraminiferal Research, Special Publication, 4, 1-349.

Cushman, J. A. 1948. Additional new foraminifera from the American Paleocene. Contributions of the Cushman Laboratory for Foraminiferal Research, 24, 43-45.

Cushman, J. A. \& Parker, F. L. 1935. Some American Cretaceous Buliminas. Contributions of the Cushman Laboratory for Foraminiferal Research, 11, 96-101.

Cushman, J. A. \& Parker, F. L. 1936. Some American Eocene Buliminas. Contributions of the Cushman Laboratory for Foraminiferal Research, 12, 39-45.

Culver, S. J. 2003. Benthic foraminifera across the Cretaceous-Tertiary (K-T) boundary: a review. Marine Micropaleontology, 47, 177-226.

Denham, C. R. \& Scotese, C. R. 1987. Terra Mobilis: a plate tectonic program for the Macintosh, versión 1.1. Geotimes, 26, Earth in Motion Technology, Austin, TX.

d'Hondt, S. 2005. Consequences of the Cretaceous/Paleogene 
mass extinction for marine ecosystems. Annual Review of Ecology, Evolution, and Systematics, 36, 295-317.

d'Hondt, S., Donaghay, P., Zachos, J. C., Luttenberg, D. \& Lindinger, M. 1998. Organic carbon fluxes and ecological recovery from the Cretaceous-Tertiary mass extinction. Science, 282, 276-279.

d'Orbigny, A. 1839. Voyage dans l'Amerique Méridionale; Foraminifères. P. Bertrand, Strasbourg, 5, 60.

Ehrenberg, C. G. 1854. Mikrogeologie. Leipzig, 22 pp.

Finlay, H. J. 1939. New Zealand foraminifera; key species in stratigraphy. Royal Society of New Zealand, 69, 325.

Fisher, M. J. 1969. Benthonic foraminifera from the Maestrichtian Chalk of Galicia Bank, West of Spain. Palaeontology, 12, 189-200.

Fontanier, C., Jorissen, F. J., Licari, L., Alexandre, A., Anschutz, P. \& Carbonel, P. 2002. Live benthic foraminiferal faunas from the Bay of Biscay: faunal density, composition and microhabitats. Deep-Sea Research I, 49, 751-785.

Gardin, S. \& Monechi, S. 1998. Palaeoecological change in middle to low latitude calcareous nannoplankton at the Cretaceous/Tertiary boundary. Bulletin de la Société Géologique de France, 169, 709-723.

Gawor-Biedowa, E. 1992. Campanian and Maastrichtian Foraminifera from the Lublin Upland, Eastern Poland. Palaeontologica Polonica, 52, 1-187.

Grzybowski, J. 1898. Otwornice pokladów naftonosnych okolicy Krosna. Rozprawy Akademii Umiejetnosci w Krakowie, Wydziat Matematyczno-Przyrodniczy, Kraków, 33, 257-305.

Hagenow, F. von. 1842. Monographie der Rügen'schen Kreide-Versteinerungen; Abtheilung III- Mollusken. Neues Jahrbuch für Mineralogie, Geognosie, Geologie und Petrefakten-Kunde, 528-575.

Hildebrand, A. R., Penfield, G. T., Kring, D. A., Pilkington, M., Camargo, A. Z., Jacobsen, S. B. \& Boynton, W. V. 1991. Chicxulub Crater: a possible Cretaceous/Tertiary boundary impact crater on the Yucatan Peninsula, Mexico. Geology, 19, 867-871.

Hollis, C. J. 2003. The Cretaceous/Tertiary boundary event in New Zealand: profiling mass extinction. New Zealand Journal of Geology and Geophysics, 46, 307-321.

Hsü, K. J. \& McKenzie, J. A. 1985. A “strangelove” ocean in the earliest Tertiary. In: Geophysical Monographs 32: The Carbon Cycle and Atmospheric $\mathrm{CO}_{2}$; Natural Variations Archean to Present (eds. E. T. Sundquist \& W. S. Broecker). AGU, Washington D.C., 487-492.

Hsü, K. J., He, Q., McKenzie, J., Weissert, H., Perch-Nielsen, K., Oberhaensli, H., Kelts, K., LaBrecque, J., Tauxe, L., Kraehenbuehl, U., Percival, S. F., Wright, R., Karpoff, A. M., Petersen, N., Tucker, P., Poore, R. Z., Gombos, A., Pisciotto, K., Carman, M. F. Jr. \& Schreiber, E. 1982. Mass mortality and its environmental and evolutionary consequences. Science, 216, 249-256.

Huber, B. T., MacLeod, K. G. \& Norris, R. D. 2002. Abrupt extinction and subsequent reworking of Cretaceous planktonic foraminifera across the Cretaceous-Tertiary boundary: Evidence from the subtropical North Atlantic.
In: Catastrophic Events and Mass Extinctions: Impacts and Beyond (eds. C. Koeberl \& K. G. MacLeod). Geological Society of America Special Paper, 356, 227-289.

Jones, R. W. \& Charnock, M. A. 1985. "Morphogroups" of agglutinated foraminifera. Their life positions and feeding habits and potential applicability in (Paleo)Ecological studies. Revue de Palèobiologie, 4, 311-320.

Jones, T. R. \& Parker, W. K. 1860. On the rhizopodal fauna of the Mediterranean compared with that of the Italian and some Tertiary deposits. Quaterly Journal of the Geological Society of London, 16, 292-307.

Jorissen, F. J., Stigter, H. C. \& Widmark, J. G. V. 1995. A conceptual model explaining benthic foraminiferal microhabitats. Marine Micropaleontology, 26, 3-15.

Jorissen, F. J., Fontanier, C. \& Thomas, E. 2007. Paleoceanographical proxies based on deep-sea benthic foraminiferal assemblage characteristics. In: Proxies in Late Cenozoic Paleoceanography (Pt. 2): Biological tracers and biomarkers (eds. C. Hillaire-Marcel \& A. de Vernal). Elsevier, Amsterdam, 263-325.

Kaiho, K. \& Lamolda, M. A. 1999. Catastrophic extinction of planktonic foraminifera at the Cretaceous/Tertiary boundary evidenced by carbon and oxygen isotopes at Caravaca, Spain. Geology, 37, 355-358.

Kaiho, K., Kajiwara, Y., Tazaki, K., Ueshima, M., Takeda, N., Kawahata, H., Arinobu, T., Ishiwatari, R., Kirai, A. \& Lamolda, M. A. 1999. Oceanic primary productivity and dissolved oxygen levels at the Cretaceous/Tertiary boundary: their decrease, subsequent warming, and recovery. Paleoceanography, 14, 511-524.

Kajiwara, Y. \& Kaiho, K. 1992. Oceanic anoxia at the Cretaceous-Tertiary boundary supported by the sulfur isotope record. Palaeogeography, Palaeoclimatology, Palaeoecology, 99, 151-162.

Keller, G. 1989a. Extended Cretaceous/Tertiary boundary extinctions and delayed population changes in planktonic foraminifera from Brazos River, Texas. Paleoceanography, 4, 287-332.

Keller, G. 1989b. Extended period of extinctions across the Cretaceous/Tertiary boundary in planktonic foraminifera of continental shelf sections: Implications for impact and volcanism theories. Geological Society of America Bulletin, 101, 1408-1419.

Keller, G. 2003. Biotic effects of impacts and volcanism. Earth and Planetary Science Letters, 215, 249-264.

Keller, G. \& Stinnesbeck, W. 1996. Sea-Level Changes, Clastic Deposits, and Megatsunamis across the Cretaceous-Tertiary Boundary. In: Cretaceous-Tertiary mass extinctions. Biotic and Environmental Changes (eds. N McLeod \& G. Keller). W.W. Norton \& Company, 415449.

Keller, G., López-Oliva, J. G., Stinnesbeck, W. \& Adatte, T. 1997. Age, stratigraphy and deposition of near-K/T siliciclastic deposits in Mexico: relation to bolide impact? Geological Society of America Bulletin, 109, 410-428.

Klaus, A., Norris, R. D., Kroon, D. \& Smit, J. 2000. Impactinduced mass wasting at the K-T boundary: Blake Nose, western North Atlantic. Geology, 28, 319-322. 
Kuhnt, W. \& Kaminski, M. A. 1993. Changes in the community structure of deep water agglutinated foraminifers across the K/T boundary in the Basque Basin (Northern Spain). Revista Española de Micropaleontología, 25, 57-92.

Kyte, F. T., Zhou, Z. \& Wasson, J. T. 1980. Siderophile-enriched sediments from the Cretaceous-Tertiary boundary. Nature, 288, 651-656.

Loeblich, A. R. \& Tappan, H. 1987. Foraminiferal genera and their classification. Van Nostrand Reinhold Company, New York, 2 vol., 1182 pp.

Marie, P. 1941. Les foraminifères de la Craie à Belemnitella mucronata du Basin de Paris. Mémoires du Musée National d'Histoire Naturelle, 12, 1-296.

Marsson, Th. 1878. Die Foraminiferen der Weissen Schreibkreide der Insel Rügen. Mittheilungen des naturwissenschaftlichen Vereins für Neu-Vorpommern und Rügen in Greifswald, 10, 115-196.

Martínez-Ruiz, F., Ortega-Huertas, M., Palomo, I. \& Barbieri, M. 1992. The geochemistry and mineralogy of the Cretaceous-Tertiary boundary at Agost (southeast Spain). Chemical Geology, 95, 265-281.

Mallory, V. S. 1959. Lower Tertiary biostratigraphy of the California Coast Ranges. American Association of Petroleum Geologists, Tulsa, Oklahoma, 1-416.

McNeil, D. H. \& Caldwell, W. G. E. 1981 Cretaceous Rocks and their foraminifera in the Manitoba escarpment. The Geological Association of Canada, Special Paper, 21, 1-439.

Molina, E., Arenillas, I. \& Arz, J. A. 1998. Mass extinction in planktic foraminifera at the Cretaceous/Tertiary boundary in subtropical and temperate latitudes. Bulletin de la Société Géologique de France, 169, 351-363.

Molina, E., Alegret, L., Arenillas, I., Arz, J.A., Martínez, F., Ortega, M., Palomo, I. \& Rodríguez, F. 2001. Field-trip guide to the Agost and Caravaca sections (Betic Cordille$\mathrm{ra}$, Spain). $6^{\text {th }}$ Workshop Esf-Impact, Granada, $72 \mathrm{pp}$.

Molina, E., Alegret, L., Arenillas, I., Arz, J. A., Gallala, N., Hardenbol, J., von Salis, K., Steutbaurt, E., Vandenberghe, N. \& Zaghbib, D. 2006. The Global Boundary Stratotype Section and Point for the base of the Danian Stage (Paleocene, Paleogene,"Tertiary”, Cenozoic) at El Kef, Tunisia: original definition and revision. Episodes, 29, 263-273.

Murray, J.W. 1991. Ecology and palaeoecology of benthic foraminifera. Elsevier, Amsterdam, 397 pp.

Nakkady, S. E. 1950. A new foraminiferal fauna from Esna Shales and Upper Cretaceous Chalk of Egypt. Journal of Paleontology, 24, 675-692.

Norris, R. D., Kroon, D., Klaus, E. et al., 1998. Proceedings of the Ocean Drilling Program Initial Reports, 171B. College Station, TX (Ocean Drilling Program).

Nuttall, W. L. F. 1930. Eocene foraminifera from Mexico. Journal of Paleontology, 4, 271-293.

Parker, W. K. \& Jones, T. R. 1862. Introduction to the study of foraminifera. Ray Society, London, $184 \mathrm{pp}$.

Peryt, D., Alegret, L. \& Molina, E. 2002. The Cretaceous/ Paleogene (K/P) boundary at Aïn Settara, Tunisia: restructuring of benthic foraminiferal assemblages. Terra Nova, 14, 101-107.
Peryt, D., Alegret, L. \& Molina, E. 2004. Agglutinated foraminifers and their response to the Cretaceous/Paleogene boundary event at Aïn Settara, Tunisia. In: Proceedings of the Sixth International Workshop on Agglutinated Foraminifera (eds. M. Bubík \& M. A. Kaminski) Grzybowski Foundation Special Publication, 8, 393-412.

Plummer, H. J. 1926. Foraminifera of the Midway Formation in Texas. University of Texas Bulletin, 2644, 1-201.

Reuss, A. E. 1844. Geognostische Skizzen aus Böhmen. C. W. Medau, Prague, 2, 1-304.

Reuss, A. E. 1845. Die Versteinerungen der böhmischen Kreideformation, Erste Abtheilung. Schweizerbart, Stuttgart, 1-57.

Reuss, A. E. 1851. Die Foraminiferen und Entomostraceen des Kreidemergels von Lemberg. Naturwissenschaftliche Abhandlungen, Wien, 4, 17-52.

Sandidge, J. R. 1932. Foraminifera from the Ripley formation of western Alabama. Journal of Paleontology, 6 , 265-287.

Sharpton, V. L., Dalrymple, G. B., Marín, L. E., Ryder, G., Schuraytz, B. C. \& Urrutia-Fucugauchi, J. 1992. New links between the Chicxulub impact structure and the Cretaceous/Tertiary boundary. Nature, 359, 819-821.

Signor, P. W. \& Lipps, J. H. 1982. Sampling bias, gradual extinction patterns, and catastrophes in the fossil record. Geological Society of America Special Paper, 190, 291298.

Smart, C. W. \& Thomas, E. 2006. The enigma of early Miocene biserial planktic foraminifera. Geology, 34, 1041-1044.

Smit, J. 1990. Meteorite impact, extinctions and the Cretaceous-Tertiary Boundary. Geologie en Mijnbouw, 69, 187-204.

Smit, J. \& Hertogen, J. 1980. An extraterrestrial event at the Cretaceous-Tertiary boundary. Nature, 285, 198-200.

Smit, J. \& Ten Kate, W. G. H. Z. 1982. Trace-element patterns at the Cretaceous-Tertiary boundary-Consequences of a large impact. Cretaceous Research, 3, 307-332.

Smit, J. \& Romein, A. J. T. 1985. A sequence of events across the Cretaceous-Tertiary boundary. Earth and Planetary Science Letters, 74, 155-170.

Smit, J., Roep, Th. B., Alvarez, W., Montanari, A., Claeys, P., Grajales-Nishimura, J. M. \& Bermudez, J. 1996. Coarsegrained, clastic sandtone complex at the Cretaceous/Tertiary boundary around the Gulf of Mexico: Deposition by tsunami waves induced by the Chicxulub impact? Geological Society of America Special Paper, 307, 151-182.

Soria, A. R., Liesa-Carrera, C. L., Mata, M. P., Arz, J. A., Alegret, L., Arenillas, I. \& Meléndez, A. 2001. Slumping and a sandbar deposit at the Cretaceous/Tertiary in the El Tecolote sector (northeastern Mexico): An impact induced sediment gravity flow. Geology, 29, 231-234.

Thomas, E. 1990a. Late Cretaceous through Neogene deepsea benthic foraminifers (Maud Rise, Weddell Sea, Antarctica). Proceedings Ocean Drilling Program, Scientific Results, 113, 571-594.

Thomas, E. 1990b. Late Cretaceous-early Eocene mass extinctions in the deep sea. In: Global Catastrophes in Earth 
History (eds. V. L. Sharpton \& P. D. Ward). Geological Society of America Special Publication, 247, 481-495.

Thomas, E., Zachos, J. C. \& Bralower, T. J. 2000. Deep-Sea Environments on a Warm Earth: latest Paleocene-early Eocene. In: Warm Climates in Earth History (eds. B. Huber, K. MacLeod \& S. Wing). Cambridge University Press, 132-160.

Thomas, E., Schmidt, D., Alegret, L., Bernaola, G., Kroon, D., Lohmann, K., Monechi, S., Roehl, U. \& Westerhold, T. 2007. Effects of the end Cretaceous asteroid impact, Walvis Ridge, SE Atlantic Ocean. 9th International Conference on Paleoceanography ICP9, Shanghai.

Toulmin, L. D. 1941. Eocene smaller foraminifera from the Salt Mountain Limestone of Alabama. Journal of Paleontology, 15, 567-611.
Van Morkhoven, F. P. C. M., Berggren, W. A. \& Edwards, A. S. 1986. Cenozoic cosmopolitan deep-water benthic foraminifera. Bulletin du Centre des Recherchers Exploration-Production Elf-Aquitaine, 11, 1-421.

White, M. P. 1928. Some index foraminifera of the Tampico Embayment area of Mexico. Part II. Journal of Paleontology, 2, 280-317.

Wicher, C. A. 1949. On the age of the higher Upper Cretaceous of the Tampico Embayment area in Mexico, as an example of the worldwide existence of microfossils and the practical consequences arising from this. Bulletin $d u$ Mussée d'Histoire naturelle du Pays Serbe, 2, 76-105.

Manuscrito recibido: 24 de Septiembre, 2007 Manuscrito aceptado: 18 de Febrero, 2008

Apéndice I. Lista taxonómica con las referencias originales de las especies de foraminíferos bentónicos representadas en la figura 2. Taxonomic list including the original references of the benthic foraminiferal species plotted in figure 2.

Alabamina wilcoxensis Toulmin

1941 Alabamina wilcoxensis Toulmin, p. 603, lám. 81, figs. 1014; textfig. 4A-C.

Anomalinoides acutus (Plummer)

1926 Anomalina ammonoides (Reuss) var. acuta Plummer, p. 149, lám. 10, figs. 2a-c.

Anomalinoides ammonoides (Reuss)

1844 Rotalina ammonoides Reuss, p. 214.

Anomalinoides rubiginosus (Cushman)

1926 Anomalina rubiginosa Cushman, p. 607, lám. 21, figs. 6a-c.

Anomalinoides susanaensis (Browning)

1959 Cibicides susanaensis Browning (en Mallory, 1959), p. 271, lám. 32, figs. 11, 12.

Arenoturrispirillina tunisiana Peryt, Alegret \& Molina. 2004 Arenoturrispirillina tunisiana Peryt, Alegret \& Molina, p. 402, lám. 1, figs. 5-7.

Bolivinoides delicatulus Cushman 1927 Bolivinoides decorata (Jones) var. delicatula Cushman, p. 90, lám. 12, fig. 8.

Bolivinoides draco (Marsson) 1878 Bolivina draco Marsson, p. 157, lám. 3, fig. 25.

Bulimina strobila Marie

1941 Bulimina strobila Marie, p. 265, lám. 32, fig. 302.

Cibicidoides abudurbensis (Nakkady)

1950 Cibicides abudurbensis Nakkady, p. 691, lám. 90, figs. 35-38.

Cibicidoides alleni (Plummer)

1926 Truncatulina alleni Plummer, p. 144, lám. 10, fig. 4.
Cibicidoides ekblomi Brotzen

1948 Cibicidoides ekblomi Brotzen, p. 82, lám. 13, fig. 2.

Cibicidoides howelli (Toulmin)

1941 Cibicides howelli Toulmin, p. 609, lám. 82, figs. 16-18.

Cibicidoides hyphalus (Fisher)

1969 Anomalinoides hyphalus. Fisher, p. 198, fig. 3.

Cibicidoides proprius Brotzen

1948 Cibicidoides proprius Brotzen, p. 78, lám. 12, figs. 3, 4.

Cibicidoides pseudoacutus (Nakkady)

1950 Anomalina pseudoacuta Nakkady, p. 691, lám. 90, figs. 29-32.

Coryphostoma decurrens (Ehrenberg)

1854 Grammostomum? decurrens Ehrenberg, p. 22, lám. 30, fig. 17 (fide Ellis \& Messina, Cat. Foram.)

Coryphostoma incrassata (Reuss)

1851 Bolivina incrassata Reuss, p. 45, lám. 4, fig. 13.

Coryphostoma incrassata gigantea (Wicher)

1949 Bolivina incrassata Reuss, forma gigantea Wicher, p. 57, lám. 5, figs. 2-3.

Eouvigerina subsculptura McNeil \& Caldwell 1981 Eouvigerina subsculptura (nomen novum) McNeil \& Caldwell, p. 21, lám. 18, figs. 20, 21.

Gaudryina pyramidata Cushman 1926 Gaudryina laevigata Franke var. pyramidata Cushman, p. 587, lám. 16, figs. 8a-b.

Gyroidinoides beisseli (White) emend. Alegret \& Thomas, 2001

1928 Gyroidina beisseli White, p. 291-292, lám. 39, figs. 7a-c. 
Gyroidinoides depressus (Alth)

1850 Rotalina depressa Alth, p.266, lám. 13, fig. 21.

Gyroidinoides girardanus (Reuss)

1851 Rotalina girardana Reuss, p. 73, lám. 5, fig. 34.

Gyroidinoides globosus (Hagenow) emend Alegret \& Thomas, 2001

1842 Nonionina globosa Hagenow, p. 574.

Loxostomoides applinae (Plummer)

1926 Bolivina applini Plummer, p. 69, lám. 4, fig. 1.

Nuttallides truempyi (Nuttall)

1930 Eponides truempyi Nuttall, p. 287, lám. 24, figs. 9, 13, 14

Oridorsalis plummerae (Cushman)

1948 Eponides plummerae Cushman, p. 44, lám. 8, fig. 9.

Osangularia plummerae Brotzen

1940 Osangularia plummerae Brotzen, p. 30, textfig. 8.

Paralabamina lunata (Brotzen)

1948 Eponides lunata Brotzen, p. 77, lám. 10, figs. 17, 18.

Praeglobobulimina quadrata (Plummer)

1926 Bulimina quadrata Plummer, p. 72, lám. 4, figs. 4, 5.

Pseudouvigerina plummerae Cushman

1927 Pseudouvigerina plummerae Cushman, p. 115, lám. 23, fig. 8 .
Pulsiphonina prima (Plummer)

1926 Siphonina prima Plummer, p. 148, lám. 12, fig. 4.

Pyramidina prolixa (Cushman \& Parker)

1935 Bulimina prolixa Cushman \& Parker, p. 98, lám. 15, fig. 5.

Repmanina charoides (Jones \& Parker)

1860 Trochammina squamata Jones \& Parker var. charoides Jones \& Parker, p. 304.

Sitella cushmani (Sandidge)

1932 Buliminella cushmani Sandidge, p. 280, lám. 42, figs. 18-19.

Sitella fabilis (Cushman \& Parker)

1936 Buliminella fabilis Cushman \& Parker, p. 7, lám. 2, fig. 5.

Sliteria varsoviensis Gawor-Biedowa

1992 Sliteria varsoviensis Gawor-Biedowa, p. 156, lám. 33, figs. 9-13.

Spiroplectammina spectabilis (Grzybowski)

1898 Spiroplecta spectabilis Grzybowski, p. 293, lám. 12, fig. 12.

Stensioeina beccariiformis (White)

1928 Rotalia beccariiformis White, p. 287, lám. 39, figs. 2a-4c.

Tappanina selmensis (Cushman)

1933 Bolivinita selmensis Cushman, p. 58, lám. 7, figs. 3, 4.

Valvalabamina lenticula (Reuss)

1845 Rotalina lenticula Reuss, p. 35, lám. 12, fig. 17. 